INPLASY

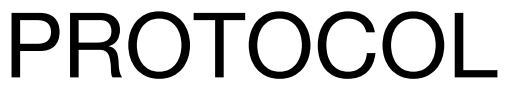

To cite: LI et al. Efficacy of cooling garment on exertional heat strain recovery in firefighters: a systematic review and meta-analysis. Inplasy protocol 2020120141. doi:

10.37766/inplasy2020.12.0141

Received: 28 December 2020

Published: 29 December 2020

Corresponding author: Jian LI

lijian@mail.dhu.edu.cn

Author Affiliation:

Donghua University

Support: Grant NO. 2232020G-08.

Review Stage at time of this submission: Data analysis.

Conflicts of interest:

None.

\section{Efficacy of cooling garment on exertional heat strain recovery in firefighters: a systematic review and meta-analysis}

\author{
LI, J1; Zhu, W2; Wang, Y3; LI, J4.
}

Review question / Objective: There is no consensus regarding the efficacy of cooling garments as a treatment for reducing heat strain. To quantify whether cooling garments mitigate exertional heat stress in randomized controlled trials (RCTs) among firefighters. Despite the apparent efficacy of cooling strategies in alleviating heat strain symptoms, not all studies found positive effects. Some suggesting a larger amount of heat decreases via cooling systems, and others suggesting that it mitigates little heat reduction compared with a control treatment, induce an additional burden, and even severely limits the individual's mobility. Thus, the effect of cooling inventions as a treatment for reducing heat strain is unclear. Condition being studied: Heat strain recovery in firefighters. The authors would like to acknowledge the financial support from the Fundamental Research Funds for the Central Universities (Grant NO. 2232020G-08). Two authors independently assessed study quality using the Cochrane Risk of Bias tool. After discussions to resolve disagreements, a consensus score was arrived at for each element of quality in each trial.

INPLASY registration number: This protocol was registered with the International Platform of Registered Systematic Review and Meta-Analysis Protocols (INPLASY) on 29 December 2020 and was last updated on 29 December 2020 (registration number INPLASY2020120141).

\section{INTRODUCTION}

Review question / Objective: There is no consensus regarding the efficacy of cooling garments as a treatment for reducing heat strain. To quantify whether cooling garments mitigate exertional heat stress in randomized controlled trials (RCTs) among firefighters. Despite the apparent efficacy of cooling strategies in alleviating heat strain symptoms, not all studies found positive effects. Some suggesting a larger amount of heat decreases via cooling systems, and others suggesting that it mitigates little heat reduction compared with a control treatment, induce an additional burden, and even severely limits the individual's mobility. Thus, the effect of 
cooling inventions as a treatment for reducing heat strain is unclear.

Rationale: Firefighters experience significant heat stress while working with heavy personal protection clothing in a hot, humid environment. Heat stress, as a rise in core temperature, can limit work capacity, impair cognitive function, and potentially induce cardiovascular strain (e.g., heat exhaustion, muscular fatigue, heat-related injuries, hyperthermia, and even death). To prevent hyperthermia and optimize performance, some cooling interventions have typically been adopted, such as garments containing phase change materials or cooled liquid, ice, gas, and air. Understanding the efficiency of the cooling invention is an important practical question for firefighters. However, the efficiency of a variety of cooling methods that differ in duration, method, and site of application on different kinds of performance has not yet been quantitatively evaluated.

Condition being studied: Heat strain recovery in firefighters. The authors would like to acknowledge the financial support from the Fundamental Research Funds for the Central Universities (Grant NO. 2232020G-08). Two authors independently assessed study quality using the Cochrane Risk of Bias tool. After discussions to resolve disagreements, a consensus score was arrived at for each element of quality in each trial.

\section{METHODS}

Search strategy: A systematic search was performed by two separate reviewers (J.L. and W.Z.) by using PubMed, Embase, and Web of Science from the earliest available date to January 2021. For other resources, we manually examined the reference list of RCTs to find additional potential eligible trials. The search strategy was carried out following the guidelines of the Preferred Reporting Items for Systematic Reviews and Meta-analyses (PRISMA), which consisted of four components included identifying, screening, qualification, and inclusion. Disagreements were solved by consensus or by a discussion with a third independent author (Y.Y.W.).

Participant or population: Firefighters.

Intervention: Cooling inventions, e.g., aircooled, liquid-cooled, phase change materials (PCM), evaporative-cooled, vacuum desiccant cooling, and hybrid cooling inventions that combine two or more cooling systems.

Comparator: No cooling.

Study designs to be included: Two reviewers separately looked at the titles and read the abstracts and filtered relevant articles to be included. The four-phase (identifying, screening, qualification and inclusion) method identified were used in the PRISMA report to diminish the number of primary search results. Disagreements were solved by consensus or by discussion with a third investigator.

Eligibility criteria: Eligible studies were included if they meet all the following criteria: (1) Study design: full-text English language articles of RCTs with reported data, excluding review articles, conference abstracts, and study protocols; (2) Population: healthy adult (age $\geq 18$ years) who identified as an outdoor worker or other physically active subjects; (3) Intervention: outdoor worker with the cooling invention (regardless of approach and liquid applied); (4) Comparison intervention: outdoor worker without any cooling invention.

Information sources: A systematic search was performed by two separate reviewers (J.L. and W.Z.) by using PubMed, Embase, and Web of Science from the earliest available date to January 2021. For other resources, we manually examined the reference list of RCTs to find additional potential eligible trials. The search strategy was carried out following the guidelines of the Preferred Reporting Items for Systematic Reviews and Meta-analyses (PRISMA), which consisted of four components included identifying, screening, qualification, and inclusion. 
Disagreements were solved by consensus or by a discussion with a third independent author (Y.Y.W.).

Main outcome(s): The main analyses compared the effects of cooling inventions versus control (without cooling inventions) on the following outcomes: physiological (core temperature [Tre], mean skin temperature [TSkin], heart rate [HR], oxygen uptake [VO2], sweat rate [SR], blood lactate [BLa-]), perceptual (rating of perceived exertion [RPE, 6 - 20] and thermal sensation [TS, 0.0 - 8.0]).

Additional outcome(s): Author and year; Country; Cooling strategies; Cooling Duration(min); Subjects (n); age (y); stature(cm); weight(kg); VO2max [mean \pm SD]; Environment [ ${ }^{\circ} \mathrm{C}, \% \mathrm{RH}$ ]; Protocols.

Data management: Data collection was performed independently by two reviewers using an independently extracted and cross-checked data form. The data were then merged by two authors and any discrepancies were settled through consultation with all authors. The data extracted from each study included (1) author and year; (2) country; (3) cooling strategies; (4) cooling duration; (5) participants, (6) environment, (7) protocols, and (8) main outcomes.

Quality assessment / Risk of bias analysis: Two authors independently assessed study quality using the Cochrane Risk of Bias tool. After discussions to resolve disagreements, a consensus score was arrived at for each element of quality in each trial.

Strategy of data synthesis: Inverse variance, a random-effects meta-analysis was then conducted on outcome data in Review Manager 5.3 (The Nordic Cochrane Centre, Copenhagen, Denmark). For each eligible article, the effect size was calculated by using the mean and standard deviation (SD) of change in each variable from baseline to postintervention for the cooling and control groups. When only the mean and SD were reported, the SD of the change was calculated by the square root of (SD2Baseline + SD2postintervention). SMD was calculated using the following formula: Where A separate meta-analysis was conducted for each outcome, with effect sizes classified based on the magnitude of change. Classifications included very small $(0.01-0.19)$, small (0.2-0.49), moderate (0.5-0.79), large (0.8-1.19), very large (1.2-1.99) and huge (> 2.0). Within each Forest plot, the included studies were grouped depending on the performance effect elicited from the training interventions (i.e. increased, decreased, no change, or unspecified effect on performance). The possibility of publication bias was assessed by plotting the effect size found in each study versus standard error in a contour-enhanced funnel plot. Funnel plot asymmetry was formally assessed using Begg and Egger's test at a significance level of $P<0.1$. The inconsistency test (12) was used to investigate the percentage of heterogeneity between the studies.

Subgroup analysis: Various cooling inventions, e.g., air-cooled, liquid-cooled, phase change materials (PCM), evaporative-cooled, vacuum desiccant cooling, and hybrid cooling inventions that combine two or more cooling systems.

Sensibility analysis: This meta-analysis employed Cochrane's risk assessment bias tool and tested each research article in six domains (1) selection bias, i.e. Random sequence generation and allocation concealment); (2) performance bias (blinding of participants and researchers); (3) detection bias (blinding of outcome assessment); (4) attrition bias (incomplete outcome data) (5) reporting bias (selective reporting) and (6) other bias. I summarized the results as 'low' when the criteria met the condition that is unlikely to influence the results and 'high' if vice versa. Another term, "unclear" was assigned when the data do not fit into the above two conditions.

Language: English language manuscripts.

Country(ies) involved: China. 
Other relevant information: The present systemic review and meta-analysis followed the guidelines provided by Preferred Reporting Items for Systematic Reviews and Meta-Analysis (PRISMA).

Keywords: heat stress; cooling garment; firefighter; meta-analysis; occupational health.

Dissemination plans: We plan to submit the manuscript to the Textile Research Journal.

Contributions of each author:

Author 1 - Jian LI - The author drafted the manuscript.

Email: lijian@mail.dhu.edu.cn

Author 2 - Wen ZHU - The author checked the manuscript.

Email: zz822069@163.com

Author 3 - Yunyi WANG - The author contributed to the development of the selection criteria and the risk of the bias assessment strategy.

Email: wangyunyi@dhu.edu.cn

Author 4 - Jun LI - The author read, provided feedback and approved the final manuscript, and he is the corresponding author.

Email: lijun@dhu.edu.cn 